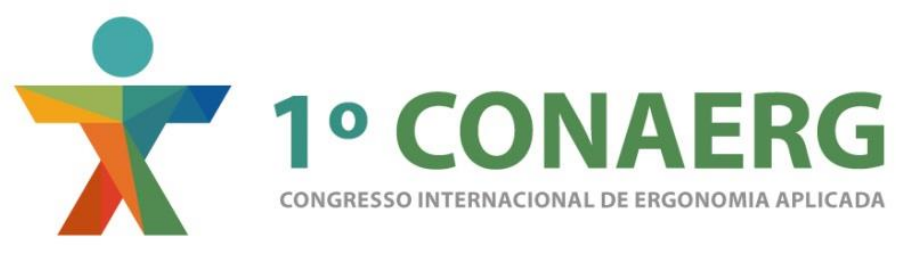

\title{
INFLUÊNCIA DO GÊNERO NO RISCO DE QUEDAS EM IDOSOS ATIVOS E SEDENTÁRIOS
}

\author{
Pedro Ferreira Reis (1); \\ Cristhiane Yoshie Tanaka (2); \\ Nayara Correa Farias (3); \\ Cristina Ribeiro da Silva (4);
}

(1) Universidade Paulista - UNIP - CESUFOZ

e-mail: fisioterapeutadotrabalho@hotmail.com

(2) Universidade Paulista - UNIP - CESUFOZ e-mail: cristhianetanaka@gmail.com

(3) Universidade Paulista - UNIP - CESUFOZ

e-mail: ncf.fisio@gmail.com

(4) Universidade Paulista - UNIP - CESUFOZ

e-mail: crisrsilva@hotmail.com

\begin{abstract}
RESUMO
O sedentarismo influência significativamente na queda em idosos. O objetivo foi avaliar a influência do gênero no risco de quedas. Participaram 100 idosos com idade entre 60 e 80 anos, sendo composto por 50 idosas e 50 idosos do município de Foz do Iguaçu, estado do Paraná, Brasil. O risco de quedas realizado através do teste Timed Up and Go. No sexo masculino o tamanho das passadas dos ativos obteve uma diferença significativa com "t" tabelado de 1,71 e o "t" calculado de 1,32. Conclui-se que a amplitude das passadas dos idosos ativos foi melhor.
\end{abstract}

Palavras-chave: Idosos; Risco de quedas; Atividade Física; Sedentarismo.

\section{ABSTRACT}

Physical inactivity significantly influence the fall in the elderly. The objective was to evaluate the influence of gender on the risk of falls. Attended 100 elderly aged 60 and 80 , comprising 50 elderly and 50 elderly in the city of Foz do Iguaçu, Paraná State, Brazil . The risk of falls accomplished through the Timed Up and Go test. In males the size of past asset obtained a significant difference " $t$ " Tabulated 1.71 and the " $t$ " calculated 1.32. It follows that the amplitude of past asset older was better. 
Keywords: Elderly ; Risk of falls ; Physical activity; Sedentary lifestyle.

\section{INTRODUÇÃO}

Há uma necessidade e interesse em relação à saúde dos idosos, a fim de tratar, prevenir, adiar e reduzir as alterações funcionais, cognitivas e fisiológicas nos processos decorrentes do envelhecimento. De acordo com o Instituto Brasileiro de Geografia e Estatística (IBGE), existe no Brasil cerca de 21 milhões de pessoas com idade igual ou superior a 60 anos, 0 que representa aproximadamente, $11 \%$ do total da população, gerando umagrandepreocupação com a saúde desses idosos (IBGE, 2013).

Segundo Siqueira et al., (2007) a ocorrência de quedas está relacionada com a inatividade física, idade avançada e maior quantidade de uso de medicamentos contínuo. Safons e Balsamo (2005) dizem que o sistema nervoso central (SNC) é mais comprometido no processo do envelhecimento, pois, além das funções biológicas internas, é o responsável pelas sensações, movimentos e funções psíquicas e não dispõe de capacidade reparadora. E que o SNC está sujeito ao envelhecimento por fatores intrínsecos (genética, metabolismo) e extrínsecos (ambiente, sedentarismo, hábitos de vida).

Observa-se uma perda progressiva de massa livre de gordura nos idosos particularmente de massa muscular esquelética, e uma concomitante redução da força muscular (GOODPASTER et al., 2008).Durante o envelhecimento há alterações que necessitam de adaptações exigindo força muscular, habilidade e amplitude de movimento (ELIOPOULOS, 2005).

Equilíbrio, força muscular, velocidade do movimento, coordenação e capacidade funcional são os benefícios do exercício físico, contribuindo assim para uma maior segurança e prevenção de quedas em pessoas da terceira idade (SPIRDUSO, 2005; BUSSE et al., 2009).

No entanto, conforme mencionam Persch et al., (2009), o treinamento de força deve ser considerado uma estratégia atrativa com o intuito de melhorar o padrão da marcha. $E$ que com os exercícios físicos leva a melhora dos níveis de força muscular nos membros inferiores. Dessa forma devem ser incentivados a prática de exercícios físicos a fim de diminuir o risco de quedas em idosos.

\section{METODOLOGIA}

Tratou-se de um estudo quantitativo do tipo descritivo desenvolvido com 100 sujeitos, sendo 50 idosas ( 25 ativas e 25 sedentárias) e 50 idosos ( 25 ativos e 25 sedentários), com faixa etária de 60 a 80 anos de idade do município de Foz do Iguaçu, Paraná, Brasil. Os dados foram coletados entre os meses de agosto a dezembro de 2014. Antes de iniciar a pesquisa, todos os participantes assinaram o termo de consentimento livre e esclarecido.

Segundo os critérios de inclusão da pesquisa foram aceitos somente os voluntários com idade entre 60 e 80 anos, do sexo feminino e masculino, sendo que os idosos ativos deveriam estar praticando atividade física regularmente com tempo mínimo de 45 minutos por 3 vezes na semana, durante os últimos 2 anos. E os idosos sedentários deveriam conseguir realizar as respiratórias, atividade de vida diária (AVD) independentemente, sua locomoção sem necessidade de órteses, não possuir diagnóstico de risco de patologias respiratórias e cardiovasculares. 
Foram excluídos os voluntários com idade inferior a 60 anos e superior a 80 anos, com limitações físicas e sensoriais absolutamente incapazes de realizar as AVD's, déficit cognitivo (com incapacidade de compreensão e execução dos movimentos através dos comandos verbais), amputação e patologias neurológicas grave.

A avaliação do risco de quedas foi através do teste Timed Up and Go (TUG), que avalia a mobilidade da funcionalidade do idoso, sendo realizado através da análise do tempo e número de passadas do idoso quando se levantar da cadeira, andar por 3 metros, retornar e sentar novamente, e quanto mais passos e tempo o idoso necessitar para concluir o trajeto, implicará em um maior risco de quedas, avaliado de acordo com o tempo, sendo inferior a 10 segundos indicará baixo risco de quedas, de 11 a 20 segundos médio risco de quedas e superior a 20 segundos indicará alto risco de quedas, iniciado por um estímulo visual "VAl" e finalizado quando o idoso senta novamente na cadeira (REIS et al., 2012; PERRACINI et al., 2002 e PODSIADLO \& RICHARDSON, 1991).

O estudo foi aprovado pelo Comitê de Ética e Pesquisa da Universidade Interativa PaulistaUNIP através da Plataforma Brasil, pelo parecer 29200314.4.0000.5512. Os dados estatísticos foram realizados através do programa Excel for Windows e Bio Estat 5.0, sendo utilizada a estatística descritiva e correlação de Pearson.

\section{RESULTADOS E DISCUSSÃO}

Sabe-se que $11 \%$ da população total do Brasil tem idade maior que 60 anos, isso aumenta a preocupação com a saúde dessas pessoas, principalmente na questão relacionada as quedas (IBGE, 2013). A ocorrência de quedas entre as idosas tem uma etiologia multifatorial que abrange fatores intrínsecos, relacionada ao próprio processo de envelhecimento e fatores extrínsecos relacionadas com o ambiente (PEREIRA et al., 2008; REIS et al., 2012).

Figura 01- Quedas no Sexo Feminino e Sexo Masculino.

\section{Quedas no Sexo Feminino (56\%)}

- 1 Queda -2 Quedas $\because 3$ Quedas

๓ 5 Quedas $₫ 8$ Quedas

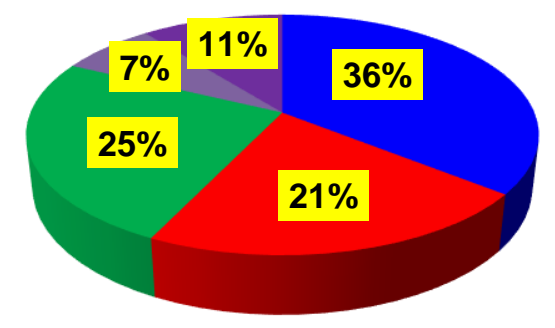

Quedas no Sexo Masculino (52\%)

- 1 Queda - 2 Quedas $=3$ Quedas

-5 Quedas $₫ 8$ Quedas

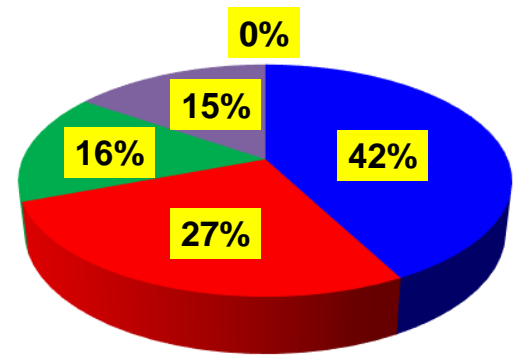

No presente estudo a população estudada foi de 100 idosos, sendo 50 de sexo feminino e 50 do sexo masculino. No sexo feminino a prevalência de queda foi de 28 idosas (56\%), dessas $10(35,71 \%)$ sofreram uma queda, $6(21,42 \%)$ duas quedas, $7(25 \%)$ três quedas, 2 $(7,14 \%)$ cinco quedas e $3(10,71 \%)$ oito quedas. E no sexo masculino a prevalência de quedas foi de 26 idosos (52\%), desses 11 (42,3\%) sofreram uma queda, 7 (26,92\%) duas 
quedas, 4 (15,38\%) três quedas e 4 (15,38\%) cinco quedas. Já Campos et al., (2013), estudou uma população idosa de 155 indivíduos e observa que 60 idosas $(38,7 \%)$ já sofreram quedas, dessas $33(55 \%)$ tiveram uma queda, 11 (18,3\%) duas quedas, $12(20 \%)$ três quedas, $1(1,1 \%)$ quatro quedas, $3(5 \%)$ cinco quedas.

$\mathrm{Na}$ presente pesquisa observou-se que os indivíduos da terceira idade do sexo feminino sofreram mais ocorrências de quedas do que o sexo masculino. Com o estudo de Gomes et al., (2009) o qual encontrou correlação dos resultados as ocorrências de quedas de duas ou mais vezes em idosas do que nos idosos. Esse resultado corrobora com a pesquisa de outros autores que justificam que devido a presença de patologias há uma maior prevalência de quedas no sexo feminino (PINHEIRO et al., 2010).

Figura 02 - Risco de Quedas do Sexo Feminino (Ativas e Sedentárias).
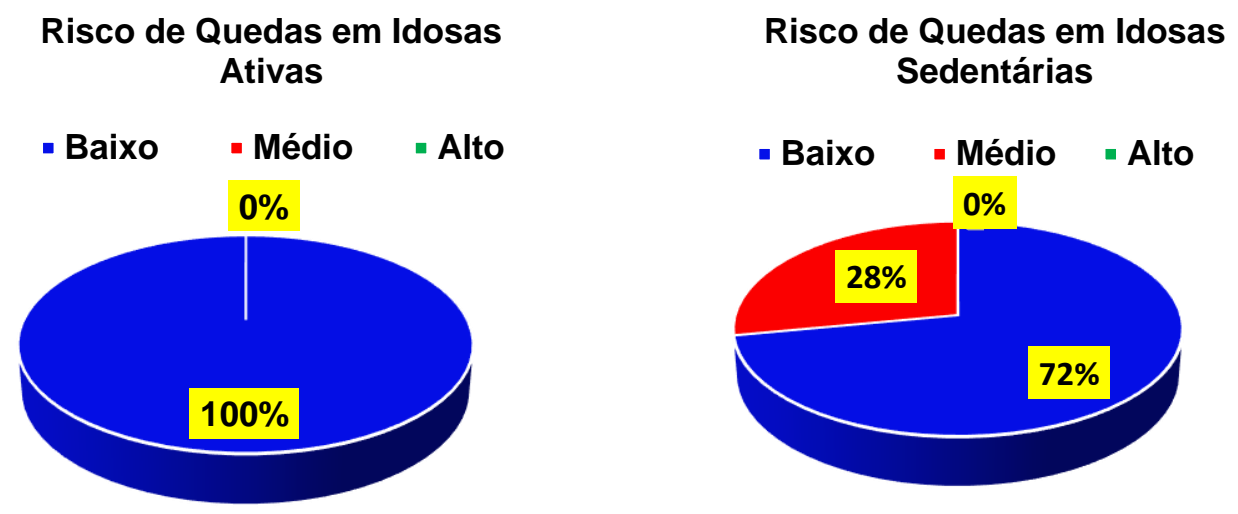

Nas idosas ativas 100\% apresentaram risco baixo de quedas, já nas idosas sedentárias somente $72 \%$ apresentaram risco baixo e $28 \%$ risco médio, porém não houve valor estatisticamente significativo.

Figura 03 - Risco de Quedas do Sexo Masculino (Ativos e Sedentários).
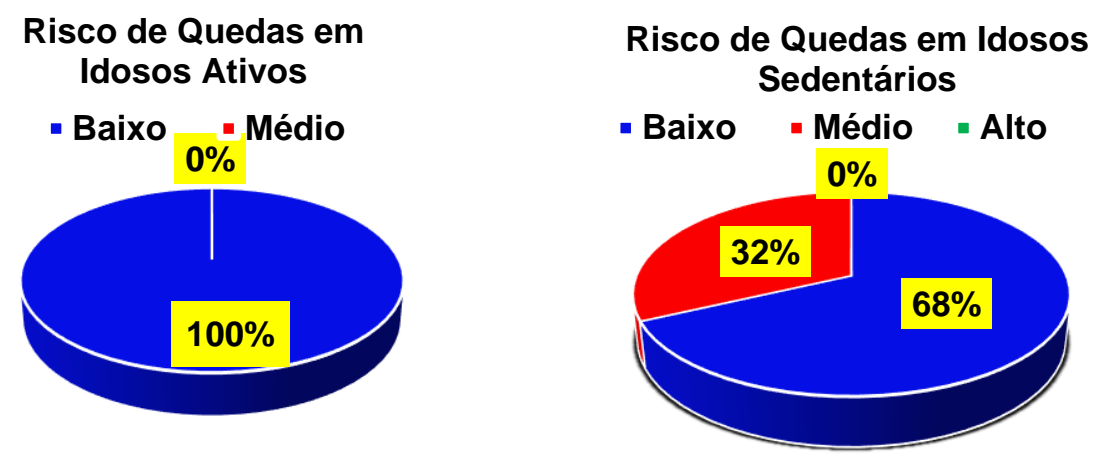

Nos idosos ativos 100\% apresentaram risco baixo de quedas, já nos idosos sedentários somente $68 \%$ apresentaram risco baixo e $32 \%$ risco médio, porém não houve significância nesses valores.

Vindo ao encontro das informações de Reis, Moro e Merino (2011), os quais obtiveram uma amostra composta por 25 idosos deambulantes, sendo 17 de sexo feminino e 8 do sexo masculino, com idade entre 67 a 80 anos, através do teste TUG concluíram que $40 \%$ desses 
participantes encontram-se grande risco de sofreram quedas, $28 \%$ risco médio e $32 \%$ com risco mínimo. Neste sentido Reis et al., (2012) alertam que o risco de quedas com idosos na faixa etária acima de 60 anos é um fator importante que deve ser considerado, principalmente pelos fisioterapeutas, arquitetos e engenheiros, em relação a eliminar os riscos ergonômicos e barreiras arquitetônicas, proporcionando assim mais segurança e conforto para os idosos realizarem suas tarefas.

Santos et al., (2011) avaliaram o nível de quedas em idosas praticantes ou não praticantes de atividade física, avaliando as participantes por meio do teste de BERG e concluiu que as idosas praticantes de atividade física têm diminuído o risco de quedas, porém apontou que este teste não é um bom instrumento para avaliar o risco de quedas em idosas ativas.

Tabela 01- Resultados das variáveis, idade, número e tamanho das passadas, tempo de execução e o nível do risco de quedas de idosas ativas e sedentárias.

\begin{tabular}{|c|c|c|}
\hline SEXO FEMININO & RESULTADO & TESTE “T” 0.05 \\
\hline Idade das ativas & $66.84 \pm 5.38$ & "T" Tabelado $(\mathrm{GL} 24)=1,71$ \\
\hline Idade das inativas & $69.68 \pm 5.92$ & "T" Calculado= -1.77 \\
\hline Número de passadas das ativas & $11.64 \pm 1.07$ & "T" Tabelado $($ GL24) = 1,71 \\
\hline Número de passadas das inativas & $12.44 \pm 2.14$ & "T" Calculado= -1.66 \\
\hline $\begin{array}{l}\text { Tamanho de passadas das ativas } \\
\text { (centímetros) }\end{array}$ & $51.97 \pm 4.91$ & "T" Tabelado $($ GL24) = 1,71 \\
\hline $\begin{array}{l}\text { Tamanho de passadas das inativas } \\
\text { (centímetros) }\end{array}$ & $49.51 \pm 7.90$ & "T" Calculado = 1.32 \\
\hline Tempo das ativas (segundos) & $8.01 \pm 0.82$ & "T" Tabelado $($ GL24) = 1,71 \\
\hline Tempo das inativas (segundos) & $9.75 \pm 1.9$ & "T" Calculado = -4.12 \\
\hline Risco de quedas nas ativas & 1 & "T" Tabelado $($ GL24) = 2,71 \\
\hline Risco de quedas nas ativas & $1.28 \pm 0.45$ & "T" Calculado= -3.01 \\
\hline
\end{tabular}

As idosas ativas apresentaram média de idade 66,84 anos $( \pm 5,38)$, e as idosas sedentárias $69,68( \pm 5,92)$, o valor médio do número de passadas das ativas $11,64( \pm 1,07)$ e das sedentárias $12,44( \pm 2,14)$, o tamanho das passadas do sexo feminino que praticam atividade física com média de $51,97 \mathrm{~cm}( \pm 4,91)$, já das que não praticam atividade física foram $49,51 \mathrm{~cm}( \pm 7,90)$, o tempo de execução do teste TUG, as ativas tiveram uma média de 8,01 segundos $( \pm 0,82)$ e as sedentárias 9,75 segundos $( \pm 1,9)$ e em relação ao risco de quedas das voluntárias ativas obteve média 1 e as inativas $1,28( \pm 0,45)$, sendo que a média 1 indica risco baixo de quedas, média 2 médio risco e média 3 alto risco de quedas.

Na tabela 02, é possível observar que a média de idade dos idosos ativos foram 66,76 $( \pm 4,88)$ e os sedentários $69,28( \pm 6,67)$, o número de passadas dos ativos $11,80( \pm 1,44)$ e os inativos $12,68( \pm 1,60)$, em relação ao tamanho das passadas dos idosos que praticam atividade física foram $51,64 \mathrm{~cm}( \pm 6,87)$ e dos que não praticam atividade física $48,01 \mathrm{~cm}$ $( \pm 5,85)$, obtendo assim a única variável com diferença significativa ("T" calculado maior que "T" tabelado 2.01) e os resultados dos voluntários ativos foram 1 (e para os voluntários sedentários $1,32( \pm 0,47)$.

Fidelis, Patrizzi e Walsh (2013) avaliaram 74 idosos, sendo 56 do sexo feminino e 18 do sexo masculino, divididos em dois grupos: Grupo Praticante de Atividade Física (GP) e o Grupo Não Praticante de Atividade Física (GNP). 
Tabela 02- Resultados das variáveis, idade, número e amanho das passadas, tempo de execução e o nível do risco de quedas de idosos ativos e sedentários.

\begin{tabular}{|c|c|c|}
\hline SEXO MASCULINO & RESULTADO & TESTE “T” 0.05 \\
\hline Idade dos ativos & $66.76 \pm 4.88$ & \multirow{2}{*}{$\begin{array}{l}\text { "T" Tabelado } \\
(G L 24)=1,71 \\
\text { "T" Calculado= } \\
-1.52\end{array}$} \\
\hline Idade dos inativos & $69.28 \pm 6.67$ & \\
\hline Número de passadas dos ativos & $11.80 \pm 1.44$ & \multirow{2}{*}{$\begin{array}{l}\text { "T" Tabelado } \\
(\text { GL24) = 1,71 } \\
\text { "T" Calculado= } \\
-2.04\end{array}$} \\
\hline Número de passadas dos inativos & $12.68 \pm 1.60$ & \\
\hline Tamanho de passadas dos ativos (centímetros) & $51.64 \pm 6.87$ & \multirow{2}{*}{$\begin{array}{l}\text { "T" Tabelado } \\
(\mathrm{GL24})=1,71 \\
\text { "T" Calculado = } 2.01\end{array}$} \\
\hline $\begin{array}{l}\begin{array}{l}\text { Tamanho de passadas dos inativos } \\
\text { (centímetros) }\end{array} \\
\end{array}$ & $48.01 \pm 5.85$ & \\
\hline Tempo dos ativos (segundos) & $8.25 \pm 0.98$ & \multirow{2}{*}{$\begin{array}{l}\text { "T" Tabelado } \\
(\text { GL24) = 1,71 } \\
\text { "T" Calculado = } \\
-4.00\end{array}$} \\
\hline Tempo dos inativos (segundos) & $10.12 \pm 02.11$ & \\
\hline Risco de quedas nos ativos & 1 & \multirow{2}{*}{$\begin{array}{l}\text { "T" Tabelado } \\
(\text { GL24) = 2,71 } \\
\text { "T" Calculado= } \\
-3.36\end{array}$} \\
\hline Risco de quedas nos ativos & $1.32 \pm 0.47$ & \\
\hline
\end{tabular}

O grupo composto por 28 mulheres e 9 homens com faixa etária entre 60 e 83 anos (média= $69,8)$ e GNP composto por 28 mulheres e 9 homens com faixa etária entre 60 e 87 anos (média=69,9). O GP realizou o teste TUG e obteve o tempo médio de 9,03 segundos, já o GNP teve média de 9.68 segundos. Portanto os resultados dos grupos (GP e GNP) foram baixo risco de quedas, não havendo também diferença significativa em relação ao gênero e atividade física no risco de quedas, porém constatou-se a melhora da flexibilidade da coluna lombar, mostrando o aumento médio de $5 \mathrm{~cm}$ em $88,1 \%$ do GP, enfatizando que a atividade física regular melhora a flexibilidade.

Assim na presente investigação científica utilizou-se o teste TUG, porém não foi encontrado diferença significativa entre as idosas ativas e sedentárias e nem diferença entre gênero, o que também foi concluído por Campos et al., (2013), que não encontrou diferença significativa estatística nos valores médios quando comparado o gênero, utilizando o TUG para analisar o risco de quedas.

Já quando se leva em conta o teste TUG para avaliação do tempo de execução, a presente pesquisa constatou que tanto as idosas quanto os idosos ativos realizaram o teste TUG em menor tempo que as idosas e idosos sedentários, o que condiz com a informação de Reis et al., (2012), Rodrigues et al., (2012) e Padoin et al., (2010) que avaliaram idosas ativas e concluíram que quanto mais ativo for a idosa, menor será o tempo para realizar o teste TUG.

Conforme Costa (2014), afirma que independentemente do tipo de treino o grupo que praticou exercício físico apresentou valores significativos melhores em relação aos níveis de 
flexibilidade na maioria das articulações, comparada ao grupo controle que não realizou exercício físico. E que possivelmente os idosos do grupo que realizou exercício físico possuem estilos de vida mais ativo, refletindo assim na amplitude de movimento (ADM).

Filho e Neto (2006) e Reis et al., (2012) afirmaram que a prática de atividade física é benéfica para evitar diversas alterações devido ao envelhecimento em nível psíquico, produz sensações de bem-estar, diminuindo a ansiedade e depressão. Com a melhora da capacidade física aumentará a autoconfiança obtendo assim uma vida ativa. Assim como Gomes e Zazá (2009), destacaram a importância da prática regular da atividade física para as idosas, que auxilia na melhora ou manutenção da saúde e a prevenção de patologias.

\section{CONCLUSÃO}

Conclui-se que na amostra pesquisada não houve diferença significativa em relação ao gênero e atividade física nos idosos, porém a amplitude da passada dos idosos do sexo masculino apresentou uma significância estatística.

Porém notou-se que das 50 idosas do sexo feminino $56 \%$ sofreram quedas, e dos 50 idosos do sexo masculino somente $52 \%$ sofreram quedas, sendo assim as idosas sofreram mais quedas comparadas ao idoso.

Assim torna-se necessário rever os métodos da atividade física dos idosos ativos em relação aos inativos. Sugerem-se novas pesquisas para verificar a fidedignidade do teste TUG com relação aos idosos de ambos os sexos, praticantes e não praticantes de atividade física, seja com uma amostra maior de participantes ou separando pelos métodos de atividade física como caminhada, academia, hidroginástica, dentre outros, atentando mais para esses pequenos detalhes dos idosos ativos para poder comparar com idosos sedentários.

\section{REFERÊNCIAS BIBLIOGRÁFICAS}

BUSSE, A.L. et al. Physical activity and cognition in elderly: a review. Dement Neuropsycol. v.3, n.3, p.204-208, 2009.

CAMPOS, K.S. et al. Os testes de equilíbrio Alcance Funcional e "Timed Up and Go" e o risco de quedas em idosos. Rev. Kairós Gerontologia. V.16, n.4, p.125-138, 2013.

COSTA, K.S. Análise de diferença dos programas de exercício físico sobre a amplitude de movimento articular de idosos de ambos os sexos. 2014. Dissertação (Mestrado de atividade física para a terceira idade). Faculdade de Desporto da Universidade do Porto ao abrigo, Porto, 2014.

ELIOPOULOS, C. Enfermagem gerontológica. Porto Alegre, Artmed, 2005.

FIDELIS, L.T; PATRIZZI, L.J; WALSH, I.A.P. Influência da prática de exercícios físicos, sobre a flexibilidade, força muscular manual e mobilidade funcional em idosos. Rev. Bras. Geriatr. Gerontol. v.16, n.1, p.109-116, 2013.

FILHO, E.; NETTO, M. Geriatria - Fundamentos clínicos e terapêuticos. São Paulo. Atheneu, 2006.

GOMES, G.A.O. et al. Comparação entre idosos que sofreram quedas segundo desempenho físico e números de ocorrências. Rev. Bras. Fisiot. v.12, n.5, p.430-437, 2009. 
GOMES, K.V.; ZAZÁ, D.C. Motivos de adesão a pratica de atividade física em idosos. Rev. Bras. De Atividade Física e Saúde. v. 14, n. 2, p.132-138, 2009

GOODPASTER, B.H.; CHOMENTOWSKI, P.; WARD, B.K. et al. Effects of physical activity on strength and skeletal muscle fat infiltration in older adults: a randomized controlled trial. Journal of Applied Physiology. v.1, n.105, p.1498-1503, 2008.

IBGE. Instituto Brasileiro de Geografia e Estatística. Indicadores populacionais. Rio de Janeiro, 2008. Disponível em: http://www.ibge.gov.br/home/estatistica/populacao/projecao da populacao/2008/projecao.p df Acesso em 10 de setembro de 2013.

PADOIN, P.G. et al. Análise comparativa entre idosos praticantes de exercícios físicos e sedentários quanto ao risco de quedas. Mundo Saúde. v.34, n.2, p.158-164, 2010.

PEREIRA, C.L.N; VOGELAERE, P, BAPTISTA, F. Role of physical activity in the prevention of falls and their consequences in the elderly. Eur Rev Aging Phys Act. v.5, n. 1, p.51-58, 2008.

PERRACINI, M. et al. Fatores associados a quedas em uma coorte de idosos residentes na comunidade. Revista de Saúde Pública. v.36, n.6, 2002.

PERSCH, L.N.; et al. Strength training improves fall-related gait kinematics in the elderly: A randomized controlled trial. Clin Biomech. v.24, n.10, p.819-825, 2009.

PINHEIRO, M.M. et al. O impacto da osteoporose no Brasil: dados Regionais das Fraturas em Homens e Mulheres Adultos. Rev. Bras. Reumatol. v.1, n.50, p.113-127, 2010.

PODSIADLO, D; RICHARDON, S. The "Timed Up and Go": a test of basic functional mobility for frail elderly persons. J Am Geriatr Soc. n.39, p. 8-142, 1991.

REIS, P.F.; MORO, A.R.P.; MERINO, E. A. D. Prevenção do risco de quedas na terceira idade: Uma abordagem no ambiente construído. In: 11ํㅡㄹ ERGDESIGN - UNIHC, 2011, Manaus: UFAM, 2011, v.1.

REIS, P.F. et al. Universal Design and Accessibility: an Approach of the Influence of Muscle Strength. Work (Reading, MA), v. 41, p. 374-379, 2012.

RODRIGUES, J.E.et al. Relação entre atividade física e equilíbrio em idosos de um grupo da terceira idade. Neurobiologia. v.1, n. 75, 2012.

SAFONS MP, BALSAMO S. Treinamento de força e envelhecimento. São Paulo: 2005.

SANTOS, G.M. et al. Valores preditivos para o risco de queda em idosos praticantes e não la de Equilíbrio de Berg. Rev. Bras. Fisioter. V.15, n.2, p.95-101, 2011.

SIQUEIRA, FV, et al. Prevalência de quedas em idosos e fatores associados. Rev Saúde Pública. v.41, n. 5, p.749-756, 2007.

SPIRDUSO, W, Dimensões Físicas do Envelhecimento. São Paulo, Manole 2005. 
